\title{
Impact of medium-scale traveling ionospheric disturbances on network real-time kinematic services: CATNET study case
}

\author{
Cristhian Camilo Timoté*, José Miguel Juan, Jaume Sanz, Guillermo González-Casado, \\ Adrià Rovira-García, and Miquel Escudero
}

Research Group on Astronomy and Geomatics (gAGE), Universitat Politècnica de Catalunya (UPC), Jordi Girona 1-3, 08034 Barcelona, Spain

Received 2 December 2019 / Accepted 9 June 2020

\begin{abstract}
Medium-scale traveling ionospheric disturbances (MSTIDs) are fluctuations in the plasma density that propagate through the upper layer of the atmosphere at velocities of approximately $100 \mathrm{~m} / \mathrm{s}$ and periods reaching some tens of minutes. Due to their wavelengths, MSTIDs can degrade the performance of differential positioning techniques, such as real-time kinematics (RTK) or network-RTK (NRTK). This paper defines a novel methodology as a tool for relating the errors in NRTK positioning based on an MSTIDs indicator using the second difference in time of the slant total electron content (STEC). The proposed methodology performs integer ambiguity resolution (IAR) on the undifferenced measurements instead of using double-differenced carrier-phase measurements, as it is usual in RTK and NRTK. Statistical tests are applied to evaluate the degradation in the position errors caused by the impacts of MSTIDs on RTK and NRTK positioning over a data set spanning one year gathered from the CATNET network; a dual-frequency network of fixed permanent GNSS receivers located at the mid-latitudes of northeastern Spain. With the development of the proposed methodology for measuring the position degradation, another results of the present research are the establishment of thresholds for the proposed MSTIDs index, which can be used to monitor the positioning solution and to warn users when the measurements are affected by MSTIDs events, relating the position error to MSTIDs that affect not only the user receivers but also of the reference receivers within the network.
\end{abstract}

Keywords: medium-scale TIDs / ionospheric disturbances / NRTK / integer ambiguity resolution

\section{Introduction}

Traveling ionospheric disturbances (TIDs) are plasma density fluctuations that propagate through the ionosphere with a broad range of velocities and frequencies. Depending on the TID characteristics, some authors (Jacobson et al., 1995) distinguish between large-scale TIDs (LSTIDs) with periods greater than 1 hour and moving faster than $0.3 \mathrm{~km} / \mathrm{s}$, and medium-scale TIDs (MSTIDs) with shorter periods (less than $1 \mathrm{~h}$ ) and slower velocities (less than $0.3 \mathrm{~km} / \mathrm{s}$ ). Although the sources of MSTIDs are not unique, MSTIDs are linked to meteorological phenomena such as neutral winds, eclipses and the solar terminator (ST), which produces atmospheric gravity waves (AGW) and manifests them as TIDs at ionospheric heights (HernándezPajares et al., 2006).

The ionosphere represents one of the major signal error sources in positioning using Global Navigation Satellite Systems (GNSS). Without any modelling, the contributions of

\footnotetext{
*Corresponding author: cristhian. timote@upc. edu
}

ionospheric errors to GNSS observables can reach up to tens of metres (Sanz et al., 2013). GNSS double-frequency receivers can eliminate up to $99.9 \%$ of the ionospheric refraction effect by implementing the ionosphere-free (IF) combination of code $\left(P_{\mathrm{IF}}=\frac{f_{1}^{2} P_{1}-f_{2}^{2} P_{2}}{f_{1}^{2}-f_{2}^{2}}\right)$ or carrier-phase $\left(L_{\mathrm{IF}}=\frac{f_{1}^{2} L_{1}-f_{2}^{2} L_{2}}{f_{1}^{2}-f_{2}^{2}}\right)$ measurements at frequencies $f_{1}$ and $f_{2}$. In contrast, single-frequency receivers must apply ionospheric models to account for the ionospheric refraction (Rovira-Garcia et al., 2019). The effects of the frequency-dependent terms (including the ionospheric refraction) can be estimated using the geometry-free (GF) combination of code $\left(P_{\mathrm{GF}}=P_{2}-P_{1}\right)$ or carrier-phase measurements ( $L_{\mathrm{GF}}=L_{1}-L_{2}$ ), which cancels out the geometric part of the measurements.

In the presence of MSTIDs, the total electron content (TEC) in the ionosphere increases by some TEC units (TECU), where 1 TECU represents an ionospheric delay of approximately $16 \mathrm{~cm}$ in the L1/E1 GNSS frequency (1575.42 MHz), which is less than the expected accuracy of the corrections provided by ionospheric models (Rovira-Garcia et al., 2016); this error has a minor impact on GNSS standard positioning services 
based on code measurements. By contrast, high-accuracy positioning services (HAS) based on carrier-phase measurements, such as the precise point positioning (PPP), real-time kinematics (RTK), or network RTK (NRTK), require a precise modelling of the ionospheric effects. Indeed, fluctuations reaching several tenths of TECUs in the ionospheric corrections can affect the positioning accuracy of HAS when a precise ionospheric modelling is needed (Hernández-Pajares et al., 2000).

In the particular case of both RTK and NRTK, dualfrequency receivers can implement the $L_{\mathrm{IF}}$ combination to account for ionospheric refraction at the cost of enlarging the noise by a factor of three compared with the noise contributed by each individual signal. This is because the difference of squared frequencies dividing the measurements in the $L_{\mathrm{IF}}$ and $P_{\mathrm{IF}}$ definition (Sanz et al., 2013). For single-frequency receivers, the ionospheric delay of GNSS signals is corrected assuming that it is identical to the ionospheric delay experienced by the closest reference receiver (RTK) or to a linear combination of the ionospheric effects experienced by the network of reference receivers (NRTK). MSTIDs can degrade the ionospheric mitigation of single-frequency users because the baselines of RTK and NRTK are tens of kilometres, which are distances comparable to the typical wavelengths of MSTIDs (Alves et al., 2001; Lachapelle \& Alves, 2002).

The present paper presents a new methodology for characterizing the impact of MSTIDs on NRTK positioning, and proposes an index as a tool to alert users about the MSTIDs effect on the GNSS user positioning. The manuscript is organized as follows: Section 1 is the current introduction. Section 2 presents the data set used and the network of NRTK stations to which the study is applied. Section 3 develops a novel methodology for creating the reference measurements from undifferenced measurements for which the carrier-phase ambiguities have been fixed. Section 4 presents the implementation of an ionosphere-free combination to obtain the reference navigation solution. Section 5 assesses the degradation of the positioning using a single frequency. Section 6 analyses the degradation of the positioning caused by MSTIDs, presents the implementation of a new index to warn RTK and NRTK users about the impacts of MSTIDs effects, and provides statistical results in order to assess the MSTIDs index performance. Finally, Section 7 summarizes the main conclusions of this work.

\section{Data: the CATNET NRTK service}

The methodology implemented in this study is developed using data from a network of sixteen permanent stations equipped with dual-frequency GNSS receivers. These stations are part of the CATNET NRTK service of the Cartographic and Geologic Institute of Catalonia (ICGC) in Spain (Talaya $\&$ Bosch, 1999). Nine stations are selected for the study in the following configuration: three of the stations are used as "service user" or "rover" receivers (PLAN, MARE, and SBAR, depicted in blue in Fig. 1), whereas the remaining six are treated as fixed reference receivers (BEUD, BELL, SONA, GARR, $L L I V$, and CASE, depicted in red in Fig. 1). Details about the implemented network are presented in Table 1 and Figure 1.

Additionally, in this study, the reference receiver GARR is used as the reference RTK receiver instead of implementing

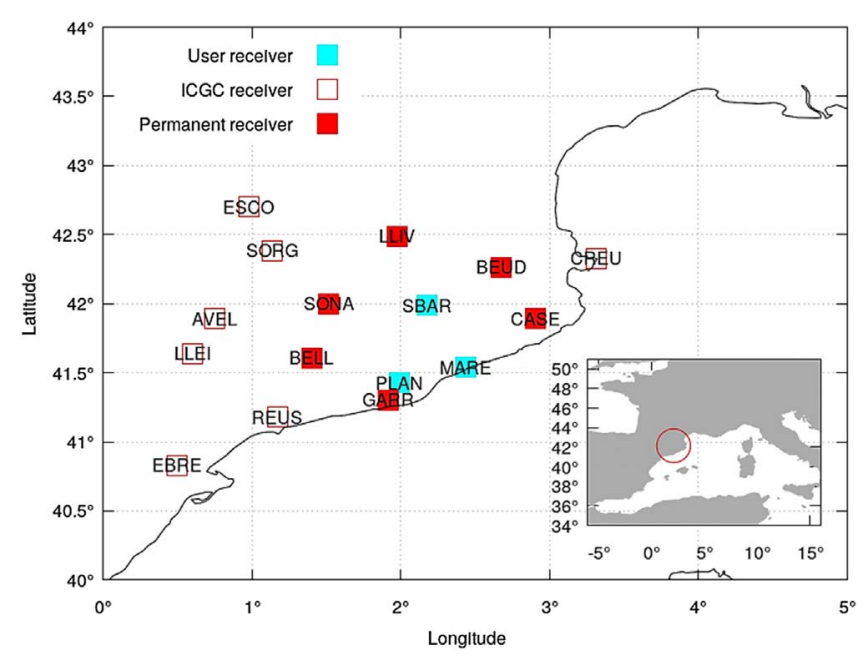

Fig. 1. CATNET NRTK network. Receivers in red: reference receivers; receivers in blue: user receivers.

the typical approach of dynamically selecting the closest reference receiver with respect to the user receiver. This approach is applied to represent the effect of the distance on the position solution for each of the three user receivers. The proposed network configuration does not represent the typical NRTK baselines between the user receiver and reference receivers, as we are implementing fixed stations as user receivers. The data set used in the present study comprises a full year of data from day of year (DoY) 200 in 2017 to DoY 200 in 2018.

\section{Methodology for obtaining unambiguous measurements in the CATNET service}

The accuracy of a HAS depends on factors such as the satellite geometry and/or the quality of the different HAS corrections (such as the ionospheric corrections). This dependency on several factors makes it difficult to separately attribute the degradation in the navigation solution to any of these factors. RTK and NRTK are based on fixing carrier phase ambiguities; therefore, one of the most relevant topics when computing the network correction is the ability to fix carrier-phase ambiguities at the network stations. To exclude this dependency, we have fixed all these carrier phase ambiguities offline. In this section, we explained how we process the data to obtain solutions with fixed carrier-phase ambiguities.

\subsection{Fixing carrier-phase ambiguities}

GNSS observables (consisting of the pseudorange $P$ and carrier phase $L$ ) emitted by a satellite $j$ and collected by a receiver $i$, at a frequency $f_{m}$ can be modelled as (Sanz et al., 2013):

$$
\begin{aligned}
& L_{m_{i}}^{j}=\rho_{i}^{j}+c\left(T_{i}-T^{j}\right)+\operatorname{Trop}_{i}^{j}-I_{m_{i}}^{j}+\lambda_{m}\left(\delta_{m i}+\delta_{m}^{j}+N_{m_{i}}^{j}\right) \\
& P_{m_{i}}^{j}=\rho_{i}^{j}+c\left(T_{i}-T^{j}\right)+\operatorname{Trop}_{i}^{j}+I_{m_{i}}^{j}+D_{m i}+D_{m}^{j}
\end{aligned}
$$

where $\rho_{i}^{j}$ stands for the geometric distance between the satellite and receiver, and $c$ is the speed of light. $T_{i}$ and $T^{j}$ are the 
Table 1. CATNET receivers' specifications.

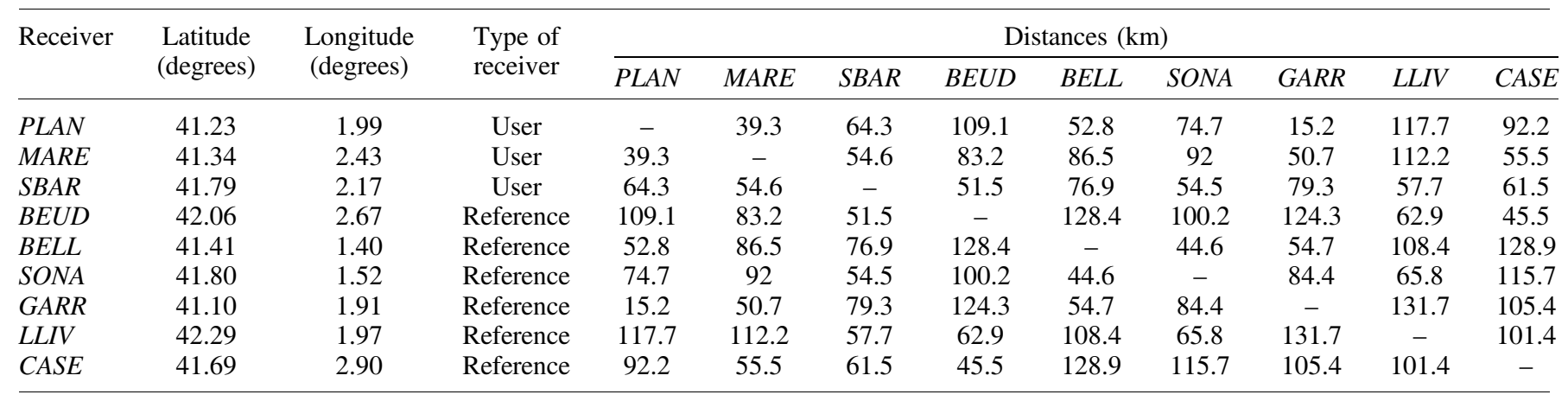

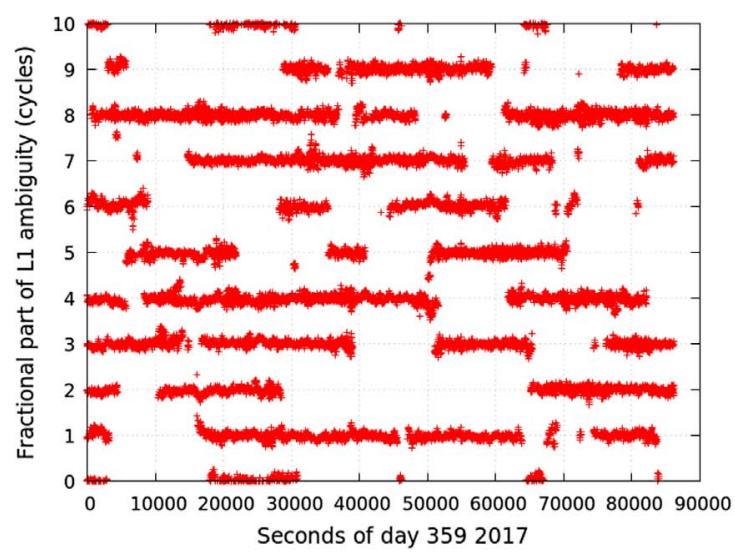

(a)

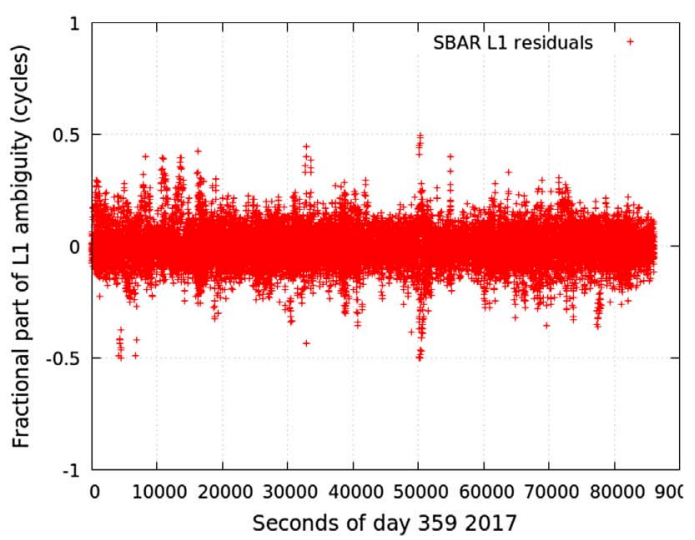

(b)

Fig. 2. Fractional part of the L1 carrier-phase ambiguities for all the satellites in view from the receivers $S B A R$ on day 359 in 2017 . The panels show the fixing-ambiguity process in which it is possible to fix the carrier-phase ambiguities with confidence.

receiver and satellite clock offsets, respectively. $\delta_{m i}, \delta_{m}^{j}, D_{m i}$, and $D_{m}^{j}$ are the instrumental delays (floated numbers), at the frequency $f_{m}$, of each of the devices. Trop ${ }_{i}^{j}$ stands for the tropospheric effect on the GNSS signal. $I_{m_{i}}^{j}$ is the ionospheric delay (advance for the phase and delay for the code measurement) proportional to $f_{m}^{-2}$. Finally, for the carrier-phase measurement, $\lambda_{m}$ represents the carrier-phase wavelength, and $N_{m_{i}}^{j}$ is the integer ambiguity. Although carrier-phase measurements $L_{m_{i}}^{j}$ are more precise than code measurements $P_{m_{i}}^{j}$, due to the unknown value of $N_{m_{i}}^{j}$, $L_{m_{i}}^{j}$ measurements are ambiguous.

In NRTK positioning, the expressions in equation (1) are simplified by forming double differences $(\Delta \nabla)$ with respect to a reference receiver and a reference satellite. One of the advantages of this technique is that the parameters depending upon a single device (either a satellite or a receiver) are cancel out, producing the following observable (Seeber, 2008):

$$
\Delta \nabla L_{m_{i}}^{j}=\Delta \nabla \rho_{i}^{j}+\Delta \nabla \operatorname{Trop}_{i}^{j}-\Delta \nabla I_{m_{i}}^{j}+\lambda_{m} \Delta \nabla N_{m_{i}}^{j} .
$$

According to equation (2), computations in a NRTK network requires carrier-phase measurements with fixed ambiguities between the stations of the network. Estimation of the exact integer value of $\Delta \nabla N_{m_{i}}^{j}$ can be achieved by means of any of the well-known method for fixing such ambiguities, such as the LAMBDA method (Teunissen, 1995). Therefore, the accuracy of the position estimates depends upon the ability to fix carrier-phase ambiguities, more precisely, on how many ambiguities are present and how fast the ambiguities are fixed (Sanz et al., 2013).

To reduce the dependency on the ability to successfully resolve ambiguities (ambiguity-fixing success ratio), we fix the integer part of the carrier-phase ambiguities of all the measurements of the undifferenced mode (i.e. before Eq. (2)). To achieve this, we have taken advantage of knowing the precise coordinates of each receiver to solve the phase biases, the so-called fractional part of the carrier-phase ambiguities (Collins et al., 2008; Ge et al., 2008), to estimate the integer part of the carrier-phase ambiguities for all the measurements in the network.

Detailed information about the whole ambiguity-fixing process can be found in (Juan et al., 2020); here, we simply present some examples of this process. As a first example of the ambiguity-fixing capability, Figure 2a depicts the actual floating values of the ambiguities (modulo 10 of the floating values) for the receiver $S B A R$ during the day 310 in 2017, illustrating that the real estimates of the ambiguities are close to the integer values (modulo 1 of the floating values). Figure $2 \mathrm{~b}$ presents 


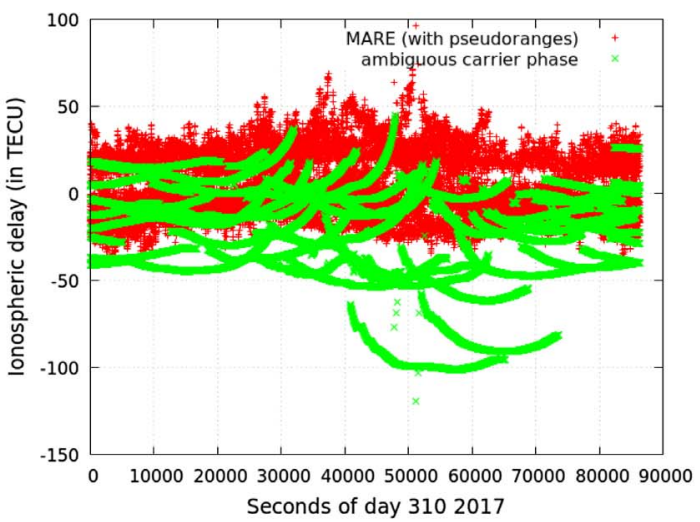

(a)

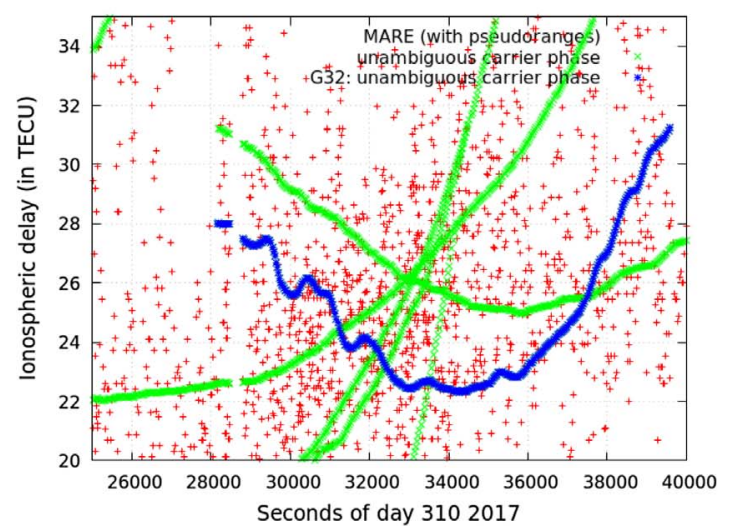

(c)

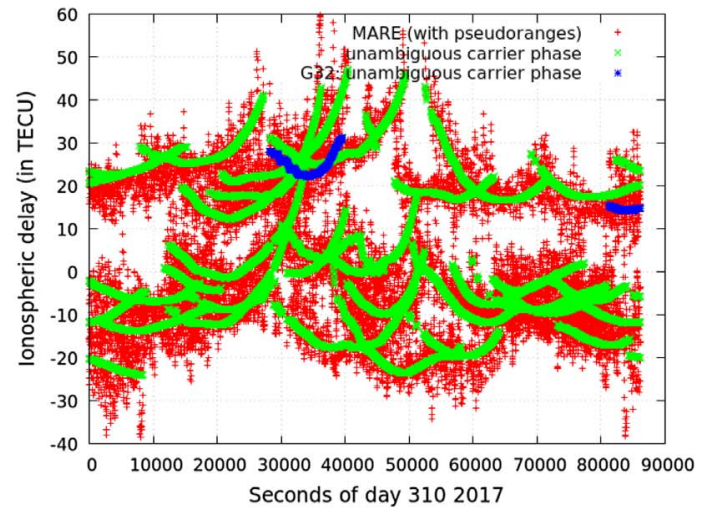

(b)

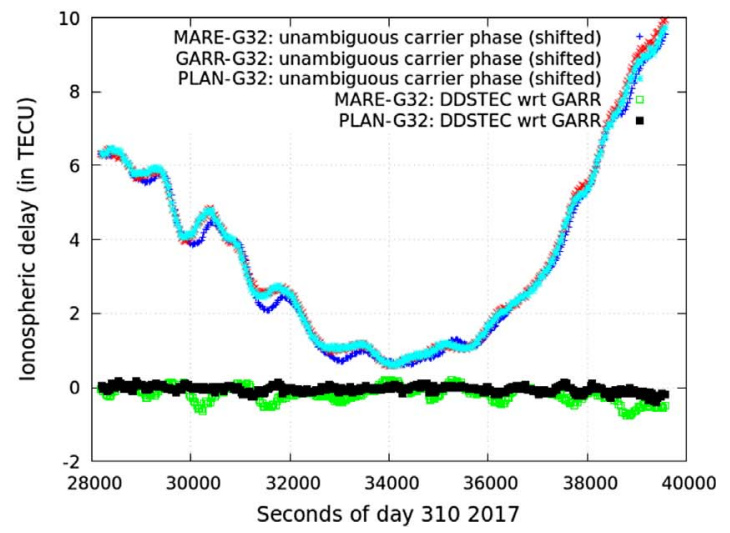

(d)

Fig. 3. Top panels: example of the ionospheric delays measured with the carrier phases (green) and pseudoranges (red) corresponding to the receiver MARE during DoY 310 in 2017. The top left panel (a) displays the raw measurements. The top right panel (b) corresponds to the measurements where the carrier-phase ambiguities have been fixed. The ionospheric delay for the satellite G32, affected by a MSTID, is highlighted in blue. The bottom left panel (c) presents a more detailed picture of the MSTID event highlighted in panel (b). The bottom right panel (d) depicts the undifferenced STEC for the satellite G32 measured by receivers MARE (blue), GARR (red), and PLAN (cyan). The double differences of the ionospheric delays with respect to GARR are also depicted in green (MARE) and black (PLAN).

how different the floating values of the carrier-phase ambiguities are from their closest integer values. The differences are typically less than 0.2 cycles, enabling the confident resolution of carrier-phase ambiguities to their integer values.

A second example showing the advantage of fixing undifferenced ambiguities can be found in Figure 3, which graphically presents a comparison between the GF combinations of the pseudoranges and carrier for the user receiver MARE. Figure $3 \mathrm{a}$ depicts the values without fixing the carrier-phase ambiguities, i.e. the raw measurements. Carrier-phase measurements are much more precise (less noisy) than pseudorange measurements. However, due to the presence of uncorrected ambiguities, the carrier phases present biases that make them less accurate than the pseudoranges. Figure $3 \mathrm{~b}$ depicts the values after fixing the carrier-phases ambiguities; after this process, the ionospheric delays obtained from the carrier phases are precise and accurate. In this panel, we highlight the ionospheric delays experienced by the GPS satellite G32 in blue to demonstrate the effect produced by a MSTID. Figure 3c presents a magnified view of the Figure $3 \mathrm{~b}$, exposing the previously mentioned disturbance. Finally, Figure $3 d$ shows the advantage of our approach (i.e., fixing the carrier-phase ambiguities in an undifferenced manner) with respect to the standard RTK method of fixing the carrier-phase ambiguities of the doubledifferenced (DD) measurements. Indeed, this panel depicts the ionospheric delays in the measurements from the satellite G32 and in those collected by the receivers MARE, GARR, and $P L A N$ in blue, red and cyan, respectively. In all three arcs, the presence of the MSTID is clear, as is the propagation of the MSTID. This is not the case for the DD measurements from $M A R E$ (green) and PLAN (black) with respect to GARR. Indeed, only the differential effects can be seen, with the amplitude of the MSTID differential effect dependent on the baseline between the receivers.

\section{Obtaining the reference navigation solution by means of the ionosphere-free combination}

Following the methodology presented in the previous section, we fix the carrier-phase ambiguities for each of the nine receivers (the user and reference receivers) selected from 
CATNET. We use these unambiguous data to compute all the navigation solutions. Note that these unambiguous measurements can be modelled as:

$$
L_{m_{i}}^{j}=\rho_{i}^{j}+c\left(T_{i}-T^{j}\right)+\operatorname{Trop}_{i}^{j}-I_{m_{i}}^{j}+\lambda_{m}\left(\delta_{m i}+\delta_{m}^{j}\right)
$$

which is mathematically equivalent to the pseudoranges $P_{m_{i}}^{j}$ model in equation (1), except for the sign of the ionospheric effect and the carrier-phase biases.

Equation (3) is the standard equation for the navigation problem, i.e. for obtaining the user position $\overrightarrow{\boldsymbol{r}}_{\boldsymbol{i}}$. After fixing carrier-phase ambiguities, there are several ways to proceed:

(i) To work with undifferenced measurements (as in Eq. (3)): in this case, one needs to know the satellite clock offsets $\left(T^{j}\right)$ and the satellite phase biases $\left(\delta_{m}^{j}\right)$, which are usually provided by several centres, such as the International GNSS Service.

(ii) For a common satellite, to make differences between the user measurements and the measurements from a nearreference receiver: in this case, one does not need the satellite parameters.

(iii) To make double differences using a common reference satellite (as in Eq. (3)): in this case, one does not need to estimate the receiver clock $\left(T_{i}\right)$.

Once ambiguities are fixed, these three techniques should provide similar results. For simplicity, let us assume we are using double differences: starting from an approximate position $\left(\overrightarrow{\boldsymbol{r}}_{o}\right)$ for the user, one can compute a value for the receiversatellite vector $\left(\overrightarrow{\boldsymbol{\rho}}_{o_{i}}^{j}\right)$. Therefore, equation (2) can be written as (Sanz et al., 2013):

$$
\Delta \nabla\left(L_{m_{i}}^{j}-\rho_{o i}^{j}\right)=\frac{\overrightarrow{\boldsymbol{\rho}}_{o_{i}}^{j}}{\rho_{o_{i}}^{j}} \Delta \overrightarrow{\boldsymbol{r}}_{i}+\Delta \nabla \operatorname{Trop}_{i}^{j}-\Delta \nabla I_{m_{i}}^{j}
$$

where obtaining the correction vector $\Delta \overrightarrow{\boldsymbol{r}}_{i}=\overrightarrow{\boldsymbol{r}}_{\boldsymbol{i}}-\overrightarrow{\boldsymbol{r}}_{o \boldsymbol{i}}$ is the basic goal of the navigation problem. This is accomplished by solving equation (4) using techniques such as least squares or Kalman filter.

Differential tropospheric corrections, $\Delta \nabla \operatorname{Trop}_{i}^{j}$, are usually estimated alongside the correction vector using a simple tropospheric model. Therefore, the main unmodelled term in equation (4) is the differential ionospheric correction $\Delta \nabla I_{m_{i}}^{j}$, which cannot be solved in equation (4). In fact, the way that $\Delta \nabla I_{m_{i}}^{j}$ is treated determines the different types of navigation solutions that we present in this work:

- Single frequency RTK solution: For each satellite, the ionospheric delays for both user and reference receiver measurements are the same $\left(\Delta \nabla I_{m_{i}}^{j}=0\right)$.

- Single frequency NRTK solution: The differential ionospheric delays, $\Delta \nabla I_{m_{i}}^{j}$ at the user position can be estimated by linearly interpolating the delays in a set of permanent receivers surrounding the user receiver.

- Ionosphere-free solution: With a receiver having two frequencies, the user can build the $L_{\mathrm{IF}}$ combination, as defined in the introduction. This combination cancels out the ionospheric effect on the GNSS signals.

From their definitions, NRTK and RTK navigation solutions will be affected by the errors in the $\Delta \nabla I_{m_{i}}^{j}$ assumptions. These errors will become larger as the baseline between the user and the reference receiver increases. In contrast, ionosphere-free measurements are unaffected by the differential ionospheric corrections. Therefore, the corresponding navigation solution $\vec{r}_{\mathrm{IF}}$ will be independent of the baseline between the user and the reference receiver.

Figure 4 depicts an example of the navigation solution obtained with the unambiguous $L_{\mathrm{IF}}$ over $24 \mathrm{~h}$ for the receiver $P L A N$, the closest user receiver with respect to the reference receiver $(15.2 \mathrm{~km}$ from the reference receiver $G A R R)$. As it can be seen, the $3 \mathrm{D}$ error in the navigation solution is usually below $10 \mathrm{~cm}$, except during some epochs presenting poor satellite geometries, i.e., when the dilution of precision (DOP) is larger than 6 (red points). 3D positioning errors better than $10 \mathrm{~cm}$ constitute the nominal performance expected for NRTK positioning (see for instance Cannon et al., 2001; Lachapelle \& Alves, 2002; Wielgosz et al., 2005).

A more general assessment of the 3D positioning error using $L_{\mathrm{IF}}$ is performed in Figure 5. This figure depicts the statistic for the three user receivers during the entire data period. This statistic is calculated by means of the complementary of the cumulative distribution function (CCDF, also represented as 1-CDF).

As depicted in Figure 5, 95\% of the cases have an error smaller than $8 \mathrm{~cm}$. This value can be established as the nominal reference value at the 95 th percentile for the $3 \mathrm{D}$ positioning error with $L_{\mathrm{IF}}$.

As expected, the three user receivers report similar statistics regardless of their distance to the reference station GARR. This is because, as mentioned above, $L_{\mathrm{IF}}$ measurements are not affected by differential ionospheric delays. Therefore, for each user receiver, this solution can be taken as the reference solution when one wants to assess the degradation in the positioning using the RTK or NRTK techniques, i.e., techniques affected by the correctness of the ionospheric assumptions.

\section{Navigation solution using either the RTK or the NRTK techniques with a single-frequency receiver}

When a user navigates solely with L1 measurements (single-frequency receivers), and therefore it is not able to build the IF combination, the navigation solutions are affected by the way in which the differential ionospheric delays are corrected.

And example of that can be seen in Figure 6, where the navigation solution using $L_{\mathrm{IF}}$, RTK and NRTK are compared. This comparison is done using the CCDFs of the navigation solutions for the three user receivers and using the three HAS techniques defined in the previous section.

The shortest baseline length of $15.2 \mathrm{~km}$ is between PLAN and GARR. In this case, as shown in Figure 6a, up to $15 \mathrm{~cm}$, the $3 \mathrm{D}$ position error using L1 (RTK or NRTK) is smaller than that using $L_{\mathrm{IF}}$. This is because the differential ionospheric effects between PLAN and GARR are quite small (see the example in Fig. 3d) and, as mentioned in the introduction, the thermal error of $L_{\mathrm{IF}}$ is amplified by a factor of three with respect that of L1 and therefore it dominates the error. On the other hand, the RTK and NRTK single-frequency solutions are quite equivalent, and the degradation of the solution with respect to that using $L_{\mathrm{IF}}$ occurs with relatively low probability (less than $1 \%$ ). 

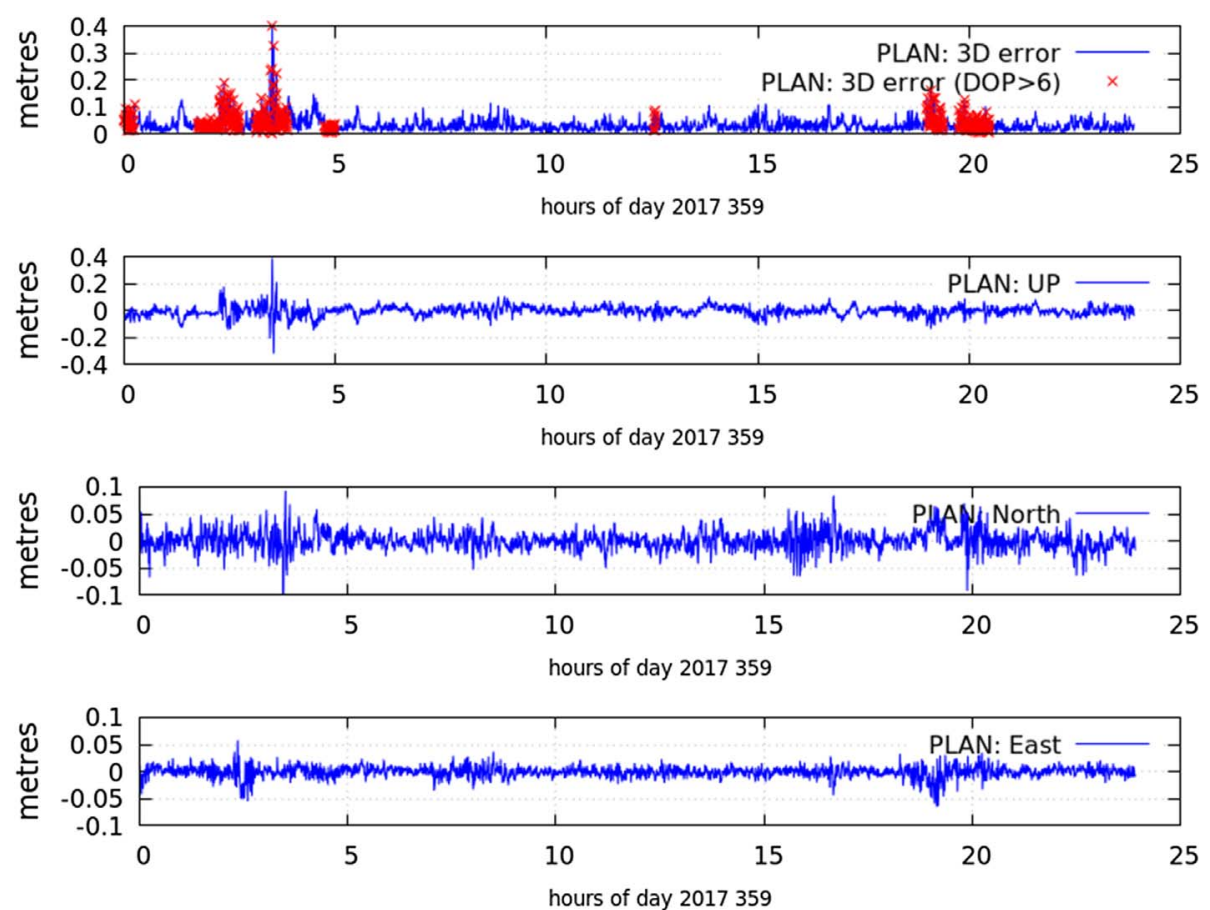

Fig. 4. Ionosphere-free combination for the navigation solution at the PLAN receiver. From top to bottom: $3 \mathrm{D}$ error, up, north, and east components.

Figure $6 \mathrm{~b}$ presents the results for MARE, located at $50.7 \mathrm{~km}$ from GARR. The differential ionospheric term in equation (4) becomes greater than in the results for PLAN, and more than $10 \%$ of the cases using L1 with RTK positioning have positioning errors larger than $8 \mathrm{~cm}$. However, if the ionospheric delay is corrected using the data from the network (NRTK), the probability of having an error larger than or equal to $8 \mathrm{~cm}$ is approximately $8 \%$. This result is not exceedingly different from the percentage found in the case with $L_{\mathrm{IF}}$ (approximately 5\%).

Finally, panel (c) of Figure 6 depicts the results for the user receiver SBAR (79.3 km from GARR). The degradation of the RTK solution increases considerably. Indeed, the probability of having an error larger than $8 \mathrm{~cm}$ in the 3D positioning reaches nearly $40 \%$, while with NRTK positioning, the probability is approximately $10 \%$, i.e., $5 \%$ more than the solution using $L_{\mathrm{IF}}$.

Taking into account the previous results, we define the 3D positioning degradation, $\delta_{3 \mathrm{D}}$, as the modulus of the difference between the single-frequency navigation (RTK or NRTK) solution $\vec{r}$ with respect to the reference solution $\vec{r}_{\mathrm{IF}}$ :

$$
\delta_{3 \mathrm{D}}=\sqrt{\left\|\vec{r}_{\mathrm{IF}}-\vec{r}\right\|^{2}} .
$$

Figure 7 presents the statistical 3D degradation by means of the $\mathrm{CCDF}$, for the three user receivers with the previously defined data set corresponding to the years 2017 and 2018. These plots confirm the results obtained and presented above. Indeed, for $S B A R$, as shown in Figure 7c, $10 \%$ of the RTK solutions present a degradation larger than $14 \mathrm{~cm}$, while less than $1 \%$ of the NRTK cases present a degradation larger than $14 \mathrm{~cm}$. However, if one compares the NRTK results for $S B A R$ with those for PLAN (Fig. 7a), one can see that the 99th percentile is less than $9 \mathrm{~cm}$ for PLAN but $12 \mathrm{~cm}$ for $S B A R$. This represents a clear

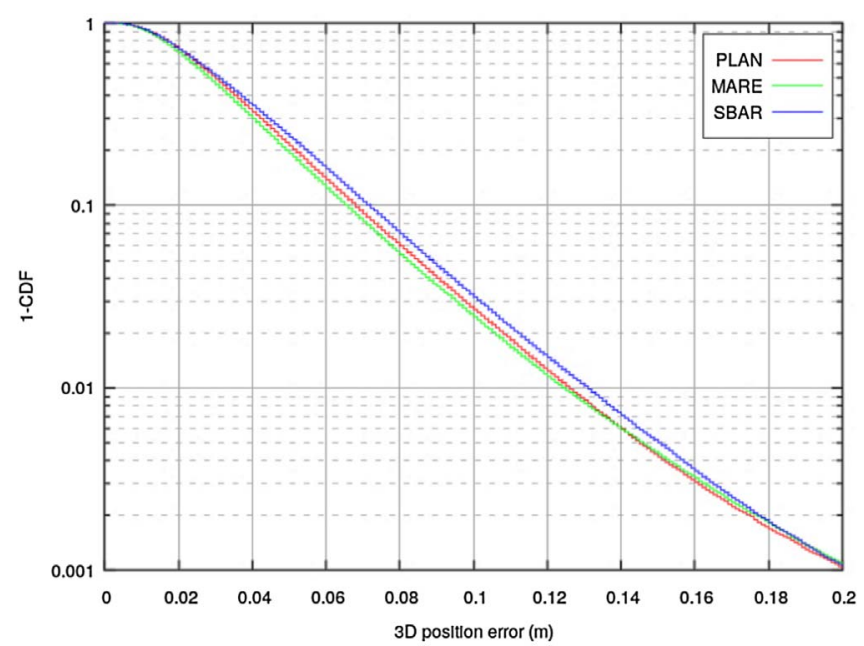

Fig. 5. Statistics for the $3 \mathrm{D}$ positioning error using the $\mathrm{IF}$ combination: the $Y$-axis depicts the probability of the 3D positioning error being greater than the value on the $X$-axis.

increase in the degradation in the NRTK solution, which is related with to the larger baseline.

\section{Effects of MSTIDs on position degradation: definition of an index linked to MSTIDs}

We have shown that the NRTK solutions are closer than RTK solutions to the dual-frequency $\left(L_{\mathrm{IF}}\right)$ solution. However, as observed in Figure 7, there is some degradation in the 


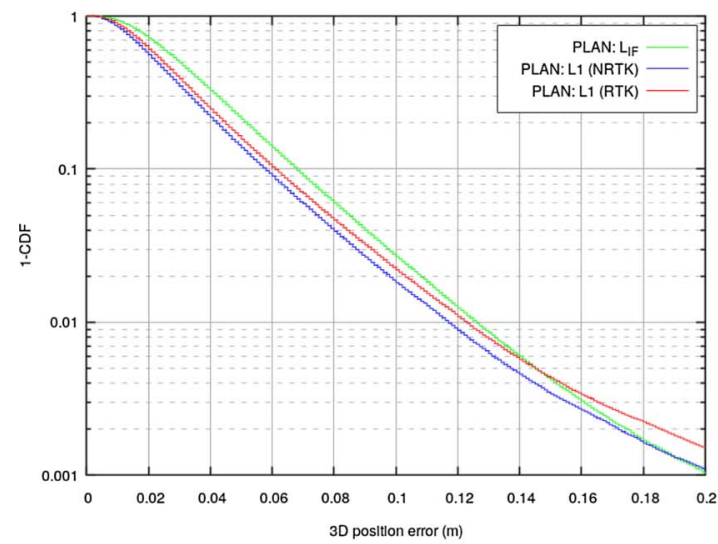

(a)

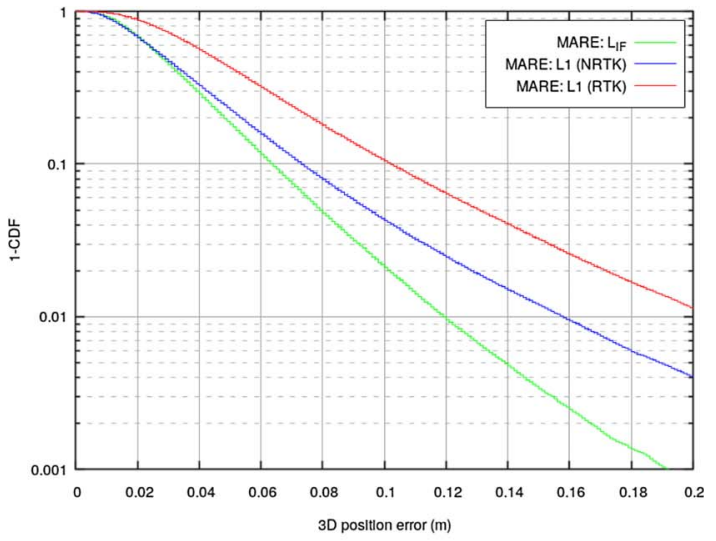

(b)

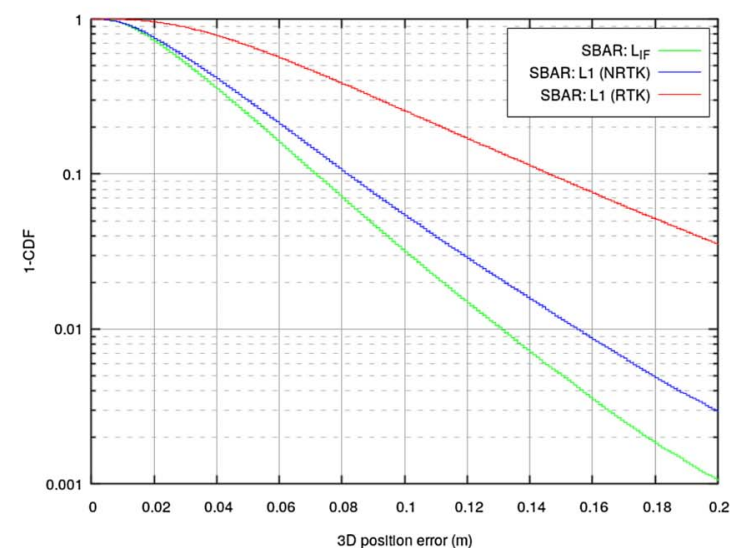

(c)

Fig. 6. Ionosphere-free $\left(L_{\mathrm{IF}}\right)$ solution in green, NRTK single-frequency (L1) solution in blue, and RTK single-frequency (L1) solution in red for three receivers: (a) PLAN, (b) MARE, and (c) SBAR.

positioning for the two receivers with the largest separation distance in the NRTK solution with respect to the IF solution. In this section, we analyse the part of the degradation in the NRTK solutions associated with the presence of MSTIDs. Indeed, NRTK positioning assumes that the differential ionospheric delays between receivers present a linear spatial behaviour. This linearity is broken by the presence of fluctuations with wavelengths (such as MSTIDs wavelengths) comparable to the network baselines. In the first subsection, we will show how MSTIDs can be detected and then propose the definition of an index linked to MSTIDs activity. In the second subsection, we will present some examples of the relationship between the position degradation and the presence of MSTIDs. Finally, in the third subsection, we will perform a statistical analysis of this relationship.

\subsection{Detecting MSTIDs and defining a MSTIDs activity index}

Hernández-Pajares et al. (2006) showed that the typical period of a MSTID is on the order of tens of minutes and further defined a method to detect such a fluctuations at this time scale by building the second difference in time $\left(\Delta^{2}\right)$ of the slant total electron content (STEC) at a given epoch $t$, defined as:

$$
\begin{aligned}
\Delta^{2} \operatorname{STEC}(t)= & 0.5 \cdot(\operatorname{STEC}(t+\tau)+\operatorname{STEC}(t-\tau)) \\
& -\operatorname{STEC}(t)
\end{aligned}
$$

where $\tau$ is set in such a way that it is optimal for detecting MSTIDs with a period of $10 \mathrm{~min}$; i.e., when $\tau$ is equal to 5 min (Hernández-Pajares et al., 2006), there is sufficient sensitivity to detect the presence of MSTIDs with periods reaching tens of minutes. Additionally, by setting $\tau$ to $5 \mathrm{~min}$, we are able to provide warnings about the presence of MSTIDs activity on a short time scale.

To quantify the activity of a MSTID, for each satellitereceiver pair, we define the MSTIDs index as the integrated amplitude over an interval $2 \tau$ (i.e., $10 \mathrm{~min}$, which results in 20 samples when the measurements are sampled at a rate of $30 \mathrm{~s}$ ):

$$
\operatorname{MSTID}_{\mathrm{IDX}}^{2}(t)=\frac{1}{20} \sum_{i=t-2 \tau}^{t}\left(M(\varepsilon) \cdot \Delta^{2} \operatorname{STEC}(i)\right)^{2}
$$

where $M(e)$ is an obliquity factor for mitigating larger values of $\Delta^{2}$ STEC $(i)$ at low elevations. 


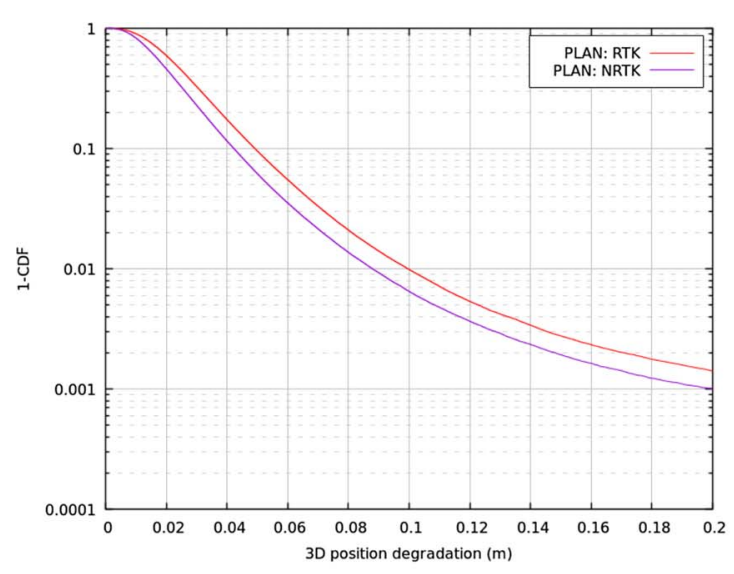

(a)

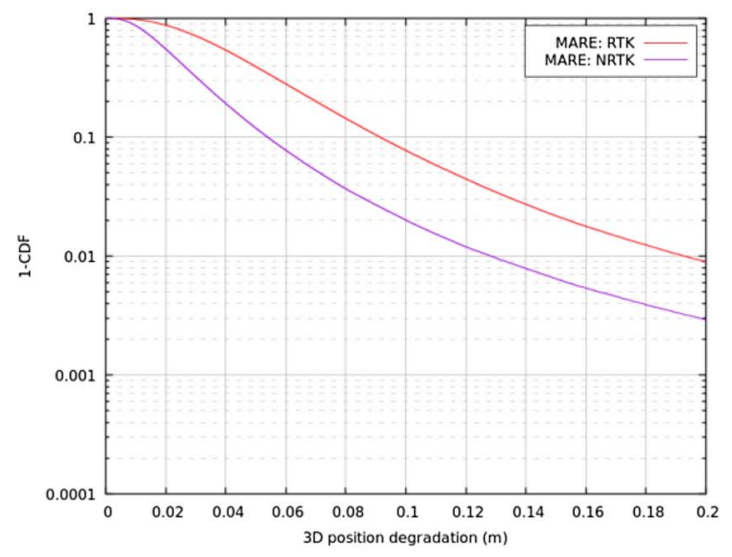

(b)

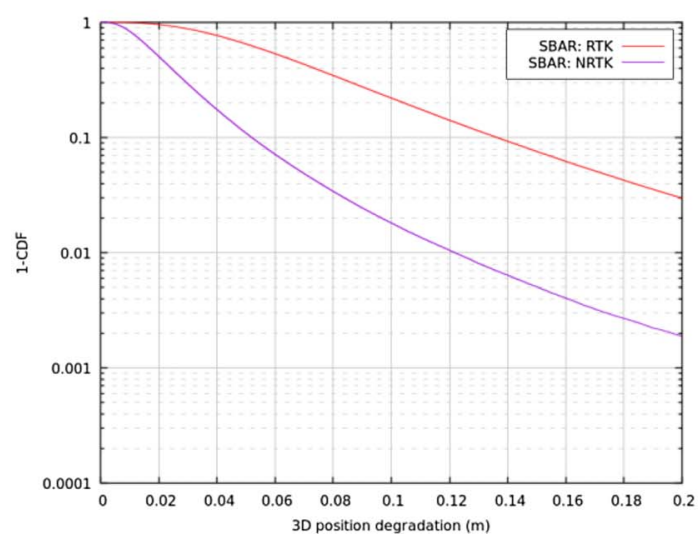

(c)

Fig. 7. CCDFs of the 3D degradation in the positioning error for the receivers (a) PLAN, (b) MARE, and (c) SBAR during the 1-year period of 2017-2018.

Figure 8 depicts the $\Delta^{2}$ STEC, as defined in equation (5), for the receivers GARR, SONA, and SBAR tracking the same GPS satellite (G01). It is clear that the presence of a MSTID (with an amplitude close to 1 TECU and a period of approximately $1000 \mathrm{~s}$ ) affects the three receivers. This STEC fluctuation clearly breaks the linearity assumption in NRTK positioning.

Figure 9 presents the results obtained after calculating

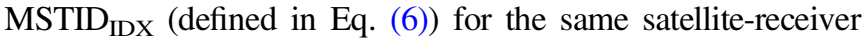
pairs as those in the example depicted in Figure 8. Certainly, it is possible to detect and isolate the MSTID event occurring at approximately $33,000 \mathrm{~s}$ of DoY 039, when MSTID IDX reaches a value of $0.5 \mathrm{TECU}$, which is $0.05 \mathrm{~m}$ for $L_{\mathrm{GF}}$ $\left(1 \mathrm{TECU}=0.105 L_{\mathrm{GF}} \mathrm{m}\right)$.

\subsection{Relationship of MSTID ${ }_{\text {IDx }}$ with the error in the ionospheric corrections}

Previous sections investigate the relation of the MSTID index with respect to the degradation in the ionospheric corrections and, consequently, in the position accuracy. Regarding the ionospheric corrections, note that this degradation is not influenced only by the MSTIDs activity during the user measurements period. Indeed, because the ionospheric delay is interpolated in NRTK positioning, any activity at any of the

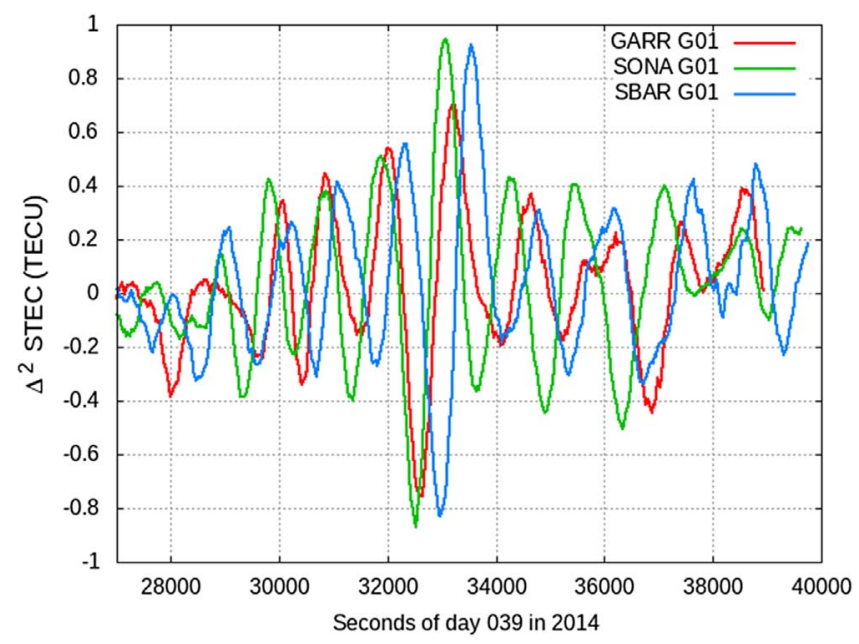

Fig. 8. MSTID effects on the second difference of the STEC for three receivers: GARR, SONA, and SBAR.

reference site contributes to the user positioning degradation. This can be seen in Figure 10, which corresponds to DoY 221 in 2017. 


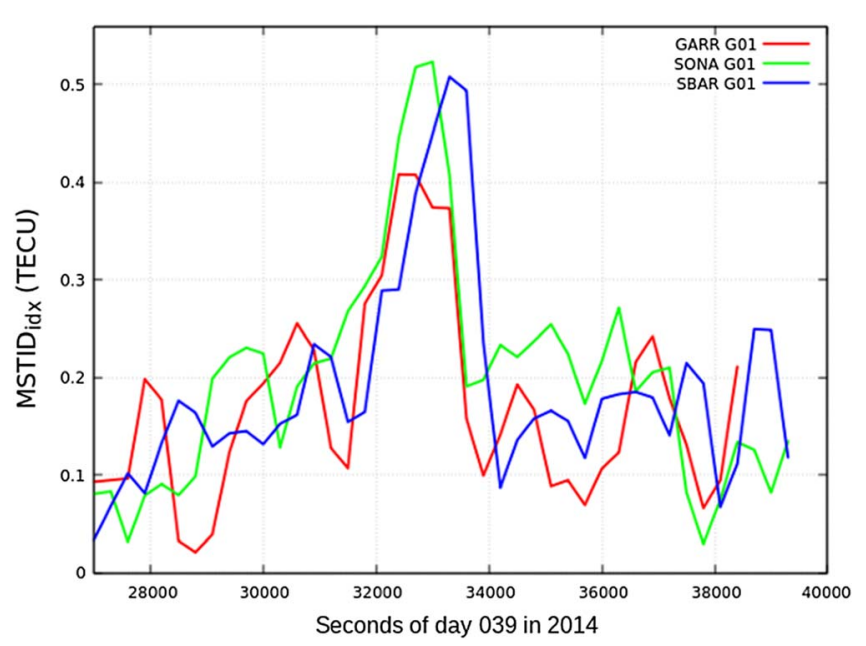

Fig. 9. MSTID index definition applied to three receivers on DoY 039 (February 6th) 2014: GARR, SONA, and SBAR.

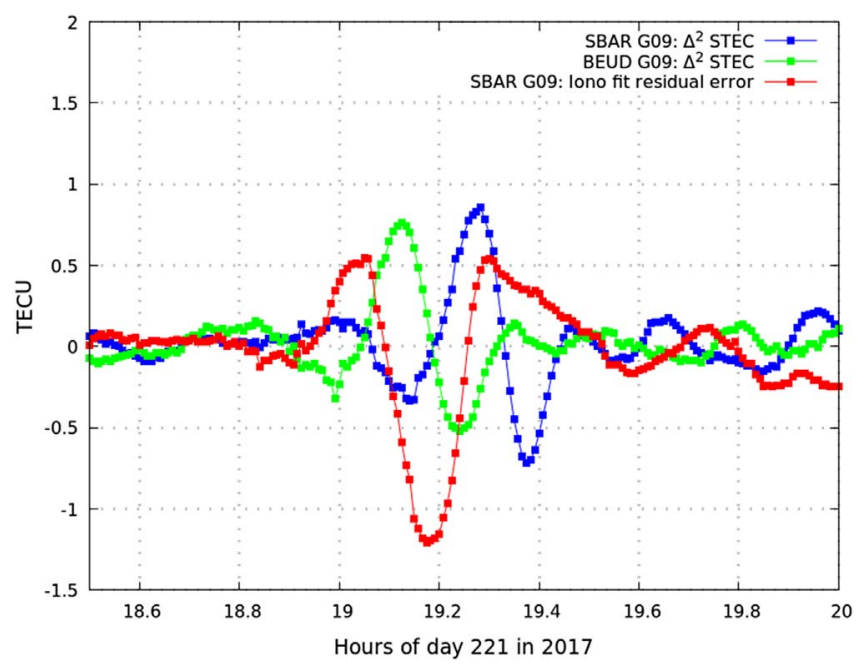

Fig. 10. $\Delta^{2}$ STEC for the user receiver $S B A R$ (blue) and reference receiver $B E U D$ (green); the error in the ionospheric correction (red) is clearly correlated with the presence of a MSTID.

Figure 10 depicts the $\Delta^{2}$ STEC experienced by measurements from the GPS satellite G09 for the user receiver $S B A R$ and for the reference receiver BEUD. The resulting error in the ionospheric correction (which is obtained by fitting a linear model from the reference sites) at $S B A R$ for the specific satellite G09 correlates with the presence of a MSTID that propagates towards the southwest. This results in a fluctuation of $\Delta^{2}$ STEC observed first at BEUD and then at SBAR. Evidently, a MSTID affecting any of the reference receivers participating in the computation of the ionospheric correction in the network contributes to the error at the user receiver.

In light of previous results, one conservative approach is to define, for each satellite in view, an MSTID index associated with the whole NRTK network. This is accomplished by taking the maximum value of the MSTID IDx experienced by any reference receiver collecting data from that satellite. Taking into account this network of MSTID $\mathrm{IDX}$ values, users can be warned about large ionospheric errors in their corrections that could

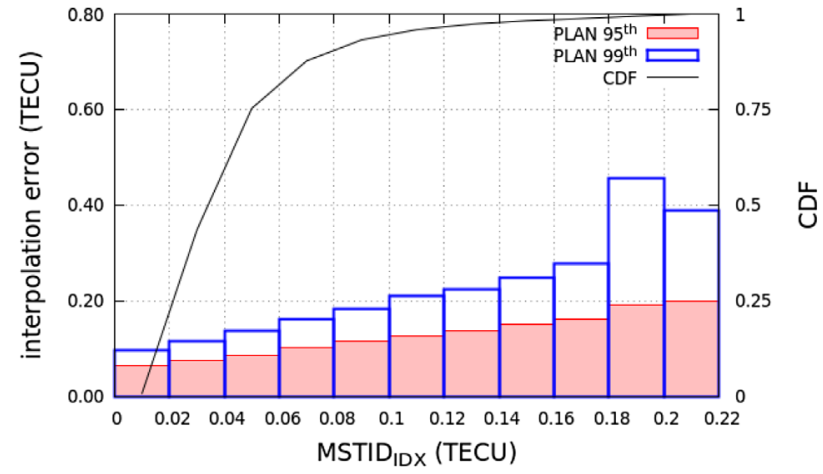

(a)

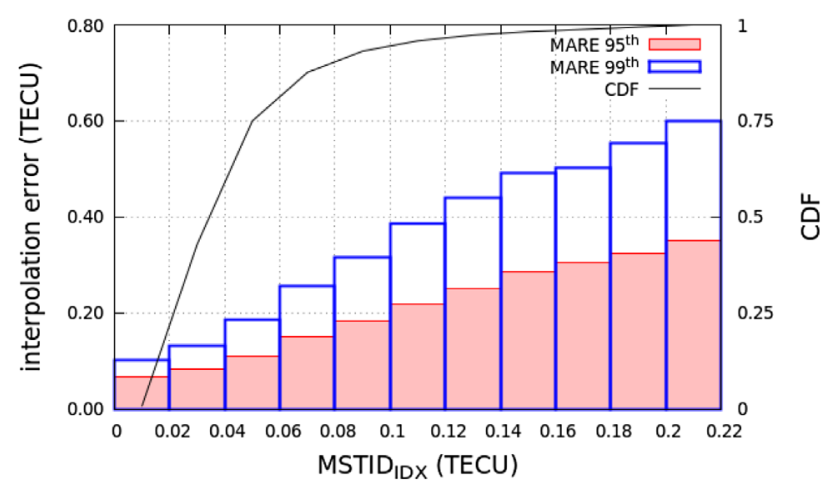

(b)

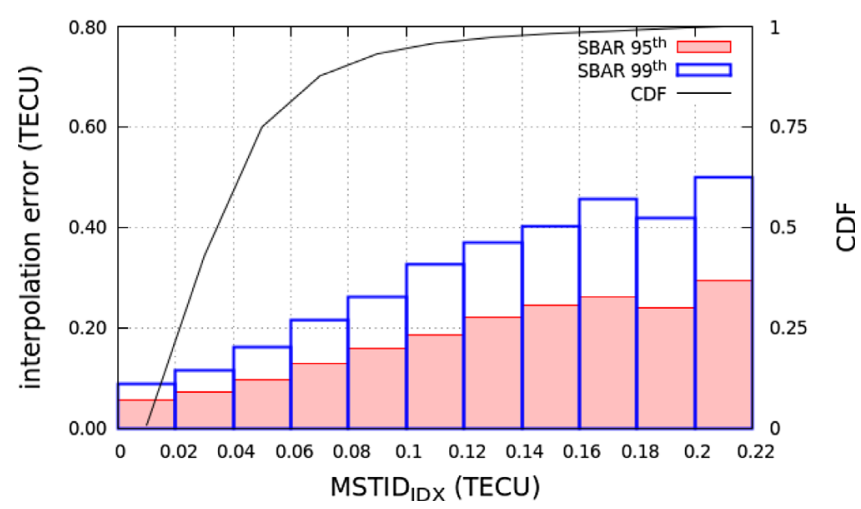

(c)

Fig. 11. Histogram of the error of the ionospheric corrections; 95th percentile (filled red boxes), 99th percentile (empty blue boxes). CDF of the ionospheric corrections (black line). The statistic is done for the three rover receivers: (a) PLAN, (b) MARE, and (c) SBAR.

affect their navigation solutions. However, the accuracy of the navigation solution depends on other factors in addition to the quality of the ionospheric corrections, such as the geometry of the observations. For that reason, we can take advantage of having undifferenced and unambiguous carrier-phase measurements to assess the errors in the ionospheric corrections at the user positions. Indeed, Figure 11 depicts the ionospheric errors during the studied period for the three user receivers. In this assessment, we consider only observations with elevation angles above $30^{\circ}$; in this way, we exclude large errors at low elevations that are not related to ionospheric activity. Each panel depicts, 
for the three user receivers, histograms of the 95th and 99th percentiles of the ionospheric errors corresponding to specific values of the MSTID IDX. As it can be seen, as the MSTID IDX $_{\text {ID }}$ increases the probability of having larger ionospheric errors also increases. Therefore, one could select some threshold value for MSTID $_{\text {IDX }}$ to exclude observations with large errors. For this purpose, we include the CDF of the overall cases in order to account for the percentage of the cases that will be filtered

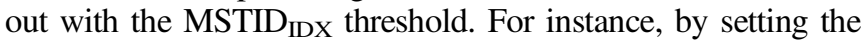
MSTID IDX $_{\text {ID }} 0.1$ TECU it is possible to guarantee that, for the three user receivers, the 95th percentile of the ionospheric corrections will be approximately less than 0.2 TECU, at the cost of excluding less than a $10 \%$ of the total measurements collected.
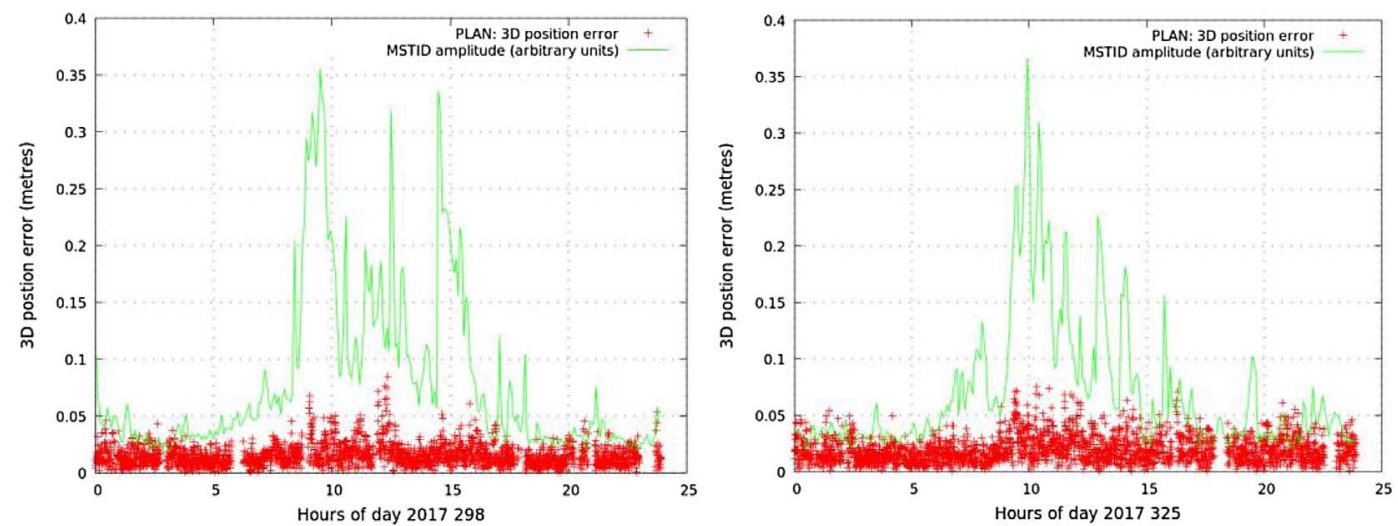

(a)
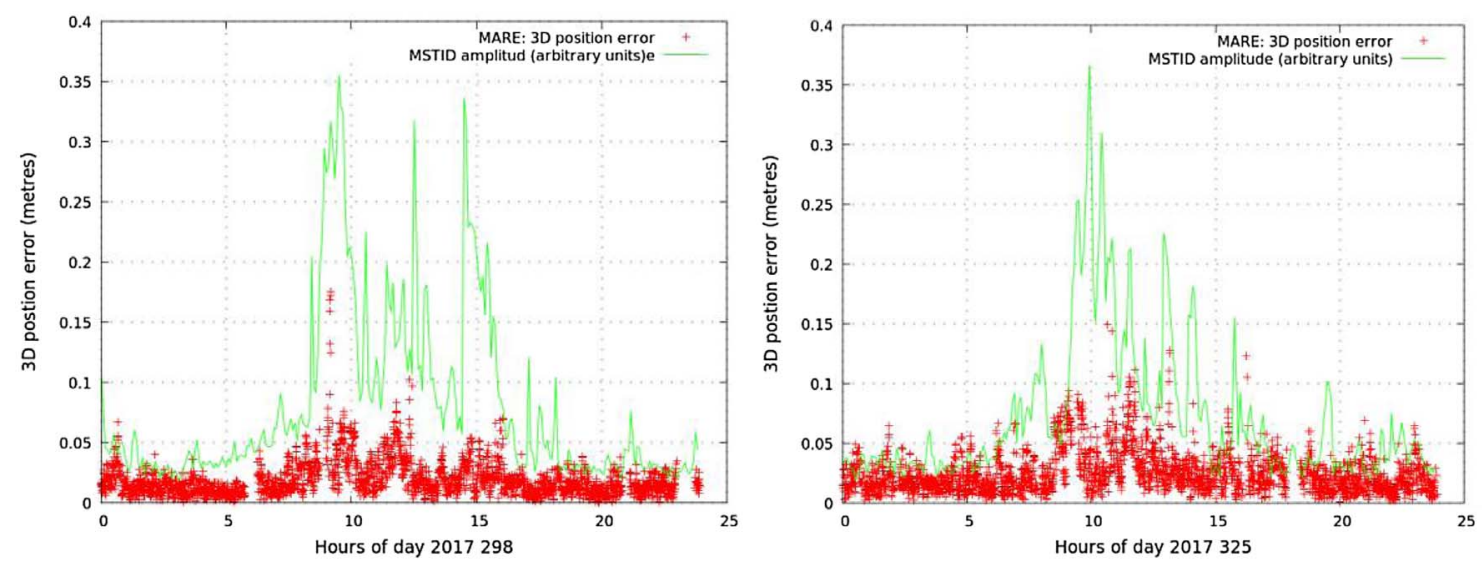

(b)
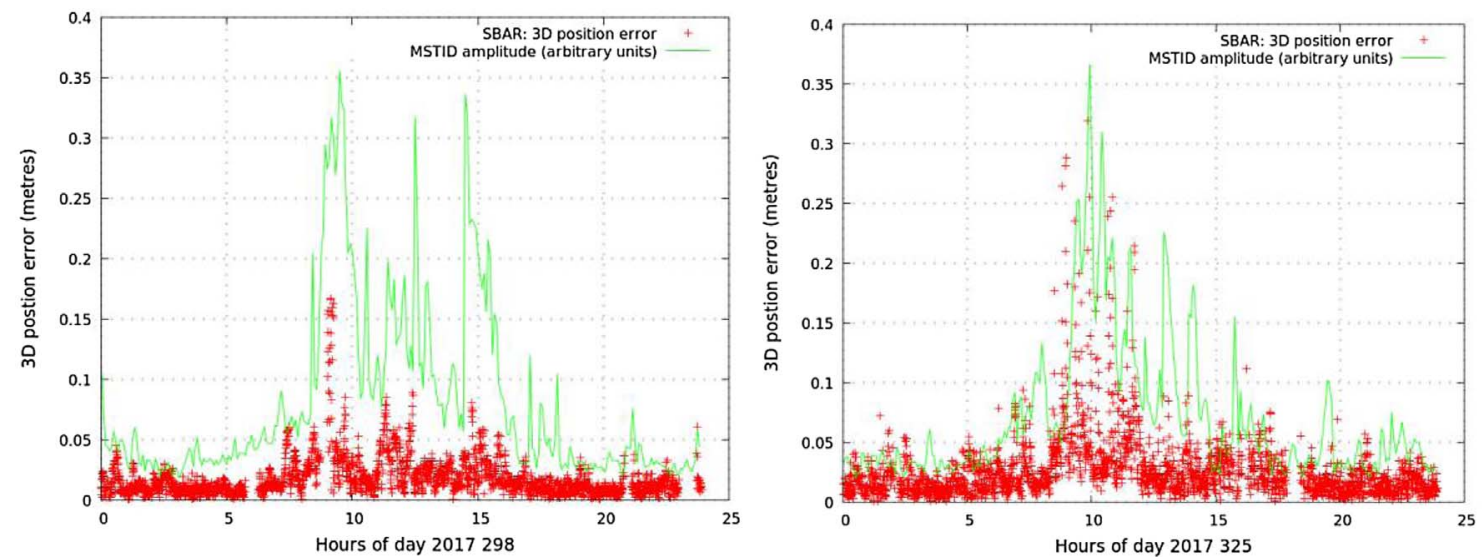

(c)

Fig. 12. 3D position errors and maximum MSTIDs amplitudes on days 298 and 325 of 2017 for the user receivers (a) PLAN, (b) MARE, and (c) $S B A R$. 


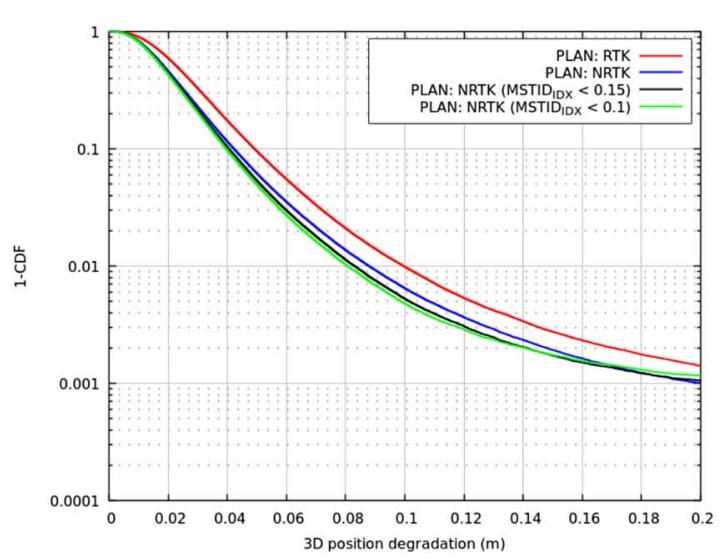

(a)

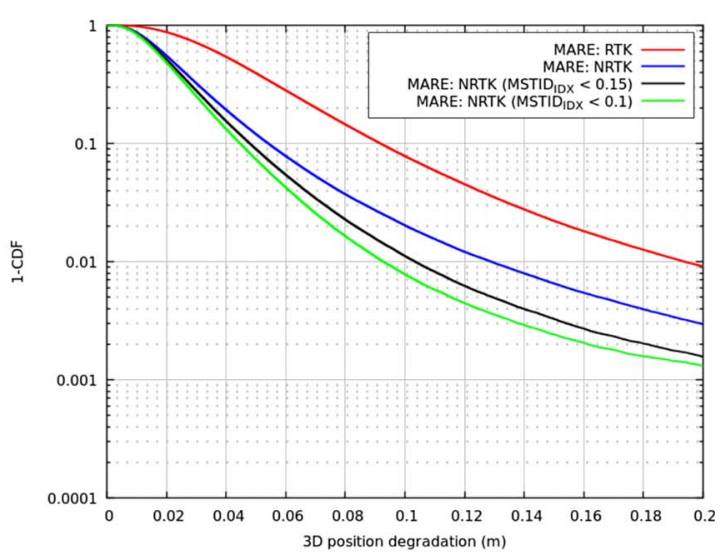

(b)

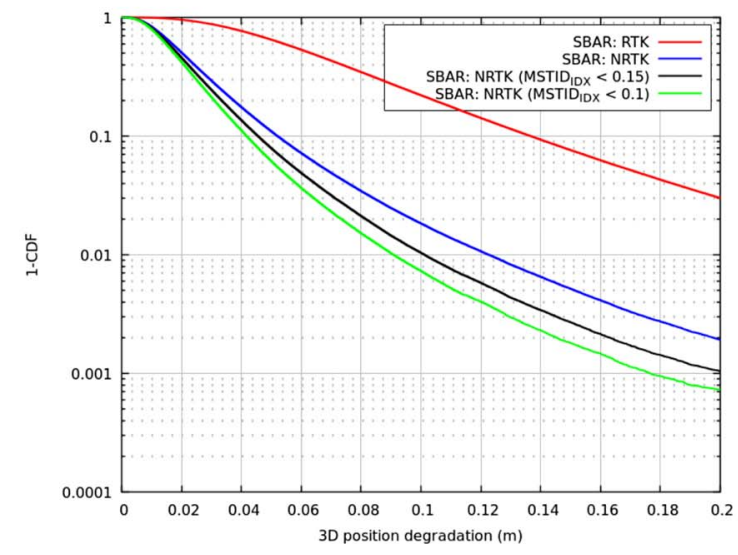

(c)

Fig. 13. 3D position degradation probabilities for user receivers: (a) PLAN, (b) MARE, and (c) SBAR. The solid lines represent the position solutions for RTK (red) and NRTK (blue) for all MSTIDs amplitudes. The NRTK solutions are also shown with MSTIDs amplitudes of less than 0.15 TECU (black) and with MSTIDs amplitudes of less than 0.10 TECU (green).

\subsection{Relationship of MSTID ${ }_{I D x}$ with the degradation in positioning}

As mentioned in previous section, for a user that needs to correct for the ionospheric effects on their observations (i.e., users with single-frequency measurements), the errors in the ionospheric corrections are translated to the position accuracy. Figure 12 presents several examples of the degradation in the 3D position errors for three stations (from top to bottom PLAN, $M A R E$, and $S B A R$ ) in contrast to the amplitude of the MSTID provided by the MSTID ${ }_{\text {IDX }}$, on the left and on the right columns respectively. The examples correspond to days 298 and 325 of 2017. A noticeable relationship exists between the degradation in the position error of the receiver and the maximum detected MSTID amplitude in the NRTK network. For a better representation of this relationship, MSTID IDX has been amplified by an arbitrary scale factor from its original amplitude value (in metres). The user receiver PLAN shows less severe degradation of the position error than the two other receivers.

For results similar to those depicted in Figure 12 encompassing the whole period of time studied in this work, readers are referred to the following public website: http://147.83.47.222/ TechTIDE_database/2017/DDD/NRTK_performance_CATNET/ Plots/, where "DDD" is the three-digit DoY.

\subsection{Statistical analysis}

We performed a statistical analysis based on the CCDFs for the position results from DoY 200 in 2017 to DoY 200 in 2018. Figure 13 provides the probability of 3D position degradation for three user receivers (PLAN, MARE, and SBAR). Figure 13 also illustrates a comparison of the degradation using RTK and NRTK positioning. Moreover, to see the relationship between the 3D position degradation and MSTIDs, the NRTK solution is also computed when:

1. MSTID IDX is lower than 0.15 TECU, for which, in SBAR, $0.1 \%$ of the cases present a degradation larger than $10 \mathrm{~cm}$ in $3 \mathrm{D}$ coordinates.

2. $\mathrm{MSTID}_{\mathrm{IDX}}$, is lower than $0.10 \mathrm{TECU}$, for which, in $S B A R, 0.2 \%$ of the cases present a degradation larger than $10 \mathrm{~cm}$ in $3 \mathrm{D}$ coordinates.

As shown in Figure 13, for the user receivers $S B A R$ and MARE, which are located farther away than PLAN, there is a noticeable reduction in the degradation error when solutions are excluded from the statistics taking into account MSTID ${ }_{\text {IDX }}$. In Tables 2-4, such a reduction in the degradation in the positioning error (by means of the 50th, 95th, and 99th percentiles) 
Table 2. NRTK position degradation (in $\mathrm{cm}$ ) for the user receiver $P L A N$ with respect to MSTID index thresholds (in TECU) by means of the 50th, 90th, and 95th percentiles.

\begin{tabular}{lccc}
\hline & $50 \%$ & $95 \%$ & $99 \%$ \\
\hline No threshold for MSTID & 1.8 & 5.3 & 8.8 \\
MSTID index less than 0.15 & 1.8 & 5.1 & 8.2 \\
MSTID index less than 0.10 & 1.7 & 4.9 & 8.0 \\
\hline
\end{tabular}

Table 3. NRTK position degradation (in $\mathrm{cm}$ ) for the user receiver $M A R E$ with respect to MSTID index thresholds (in TECU) by means of the 50th, 90th, and 95th percentiles.

\begin{tabular}{lccc}
\hline & $50 \%$ & $95 \%$ & $99 \%$ \\
\hline No threshold for MSTID & 2.1 & 7.2 & 12.8 \\
MSTID index less than 0.15 & 2.1 & 6.0 & 10.6 \\
MSTID index less than 0.10 & 2.0 & 6.0 & 9.4 \\
\hline
\end{tabular}

Table 4. NRTK position degradation (in $\mathrm{cm}$ ) for the user receiver $S B A R$ with respect to MSTID index thresholds (in TECU) by means of the 50th, 90th, and 95th percentiles.

\begin{tabular}{lccc}
\hline & $50 \%$ & $95 \%$ & $99 \%$ \\
\hline No threshold for MSTID & 2.0 & 6.7 & 12.2 \\
MSTID index less than 0.15 & 1.9 & 5.8 & 10.2 \\
MSTID index less than 0.10 & 1.8 & 5.7 & 9.3 \\
\hline
\end{tabular}

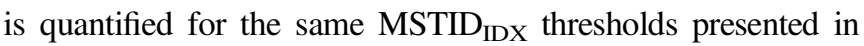
Figure 13.

From Figure 13 or the aforementioned tables, one can see that the reduction in the degradation is lower for the closest receiver, PLAN (approximately 10\%), which is almost unaffected by MSTIDs: the degradation is maintained at approximately $5 \mathrm{~cm}$ in the 95th percentile and $8 \mathrm{~cm}$ in the 99th percentile.

This is not the case for the other two receivers, where the degradation is approximately $12 \mathrm{~cm}$ in the 99th percentile when MSTID $_{\text {IDX }}$ is not taken into account. However, if the MSTI$\mathrm{D}_{\mathrm{IDX}}$ is considered, the degradation in the navigation solution is reduced up to $26 \%$ for $M A R E$ and $23 \%$ for $S B A R$. Furthermore, for the 95th percentile, SBAR and MARE reach levels of reduction similar to PLAN (the closest receiver). Therefore, MSTID $_{\text {IDX }}$ can be used as an indicator that warns users of degradation in the navigation solution.

\section{Conclusions}

The present contribution analyses the impacts of MSTIDs on a network of permanent geodetic receivers located at midlatitudes. The methodology relies on three main steps: First, as detailed in Section 3, reference measurements are obtained by applying a novel method of fixing carrier-phase ambiguities in the undifferenced measurements, avoiding the classic use of double-differenced carrier-phase measurements, where only differential MSTIDs effects can be seen. In this way, we conduct this study independent of the ambiguity fixing success rate.
Second, thanks to the previous carrier-phase ambiguity fixing, a reference navigation solution based on the ionosphere-free navigation combination $\left(L_{\mathrm{IF}}\right)$ is computed, making it possible to account for only the effects related to the ionosphere. Third, this reference navigation solution is compared with the navigation solution computed using either RTK or NRTK. In this way, we assess the impacts of the ionosphere effects on the $3 \mathrm{D}$ positioning.

The relationship between the positioning error and the MSTIDs is also shown, where the presence of an MSTID is a degrading factor for user positioning not only in RTK but also in NRTK. This degradation is related not only to the effect of the TID on the user measurements but also to the measurements of any of the reference receivers.

An MSTID index is defined and implemented as a tool to warn users about possible positioning degradation. The performance is tested with three stations located at 15, 50, and $79 \mathrm{~km}$ from the reference receiver. Using this index over a data set covering one year, we show that it is possible to obtain similar accuracies in the three baselines.

As a product related to this work, real-time estimates of MSTID $_{\text {IDX }}$, applied on more than one hundred of worldwide GNSS receivers, are computed and stored in the Warning and Mitigation Technologies for Travelling Ionospheric Disturbances Effects (TechTIDE) open repository, located at the following internet address: http://techtide.space.noa.gr/.

Acknowledgements. This work is sponsored by the European Union's Horizon 2020 research and innovation programme under grant agreement 776011 (TechTIDE project) and 797461 (NAVSCIN), and the Spanish Ministry of Science, Innovation and Universities RETOS RTI2018-094295-B-I00 (Programa Estatal de I + D + i Orientada a los Retos de la Sociedad). The authors acknowledge the use of data from the CATNET NRTK service from the Cartographic and Geologic Institute of Catalonia, Spain (Institut Cartogràfic $i$ Geologic de Catalunya - ICGC), and data from the International GNSS Service (IGS). The editor thanks Yuichi Otsuka and an anonymous reviewer for their assistance in evaluating this paper.

\section{References}

Alves P, Lachapelle G, Cannon ME, Liu J, Townsend B. 2001. Evaluation of multiple-reference DGPS RTK using a large scale network. In: Proceedings of the National Technical Meeting of the Institute of Navigation, ION NTM/2001 (January 2001, Long Beach, USA), pp. 665-671.

Cannon ME, Lachapelle G, Alves P, Fortes LP, Townsend B. 2001. GPS RTK Positioning using a regional reference network: Theory and results. In: Proceedings of the Global Navigation Satellite Systems Conference (May 2001, Seville, Spain), Global Navigation Satellite Systems.

Collins P, Lahaye F, Héroux P, Bisnath S. 2008. Precise point positioning with ambiguity resolution using the decoupled clock model. In: Proceedings of the 21st International Technical Meeting of the Satellite Division of The Institute of Navigation, ION GNSS 2008 (September 2008, Savannah, USA). pp. 1315-1322. 
Ge M, Gendt G, Rothacher M, Shi C, Liu J. 2008. Resolution of GPS carrier-phase ambiguities in precise point positioning (PPP) with daily observations. J Geodesy 82(7): 389-399. https://doi.org/ 10.1007/s00190-007-0187-4.

Hernández-Pajares M, Juan JM, Sanz J, Colombo OL. 2000. Application of ionospheric tomography to real-time GPS carrier-phase ambiguities Resolution, at scales of $400-1000 \mathrm{~km}$ and with high geomagnetic activity. Geophys Res Lett 27(13): 2009-2012. https://doi.org/10.1029/1999GL011239.

Hernández-Pajares M, Juan JM, Sanz J. 2006. Medium-scale traveling ionospheric disturbances affecting GPS measurements: Spatial and temporal analysis. J Geophys Res Space Phys 111(A07S11). https://doi.org/10.1029/2005JA011474.

Jacobson AR, Carlos RC, Massey RS, Wu G. 1995. Observations of traveling ionospheric disturbances with a satellite-beacon radio interferometer: Seasonal and local time behavior. J Geophys Res Space Phys 100(A2): 1653-1665. https://doi.org/10.1029/ 94JA02663.

Juan JM, Sanz J, Rovira-Garcia A, González-Casado G, VenturaTraveset J, Cacciapuoti L, Schoenemann E. 2020. A new approach to improve satellite clock estimates, removing the inter-day jumps. In: Proceedings of the 51st Annual Precise Time and Time Interval Systems and Applications Meeting of the US Institute of Navigation, pp. 279-301.
Lachapelle G, Alves P. 2002. Multiple reference station approach: Overview and current research. J Glob Position Syst 1(2): 133-136.

Rovira-Garcia A, Juan JM, Sanz J, González-Casado G, Ibáñez D. 2016. Accuracy of ionospheric models used in GNSS and SBAS: Methodology and analysis. J Geodesy 90(3): 229-240.

Rovira-Garcia A, Ibáñez-Segura D, Orús-Perez R, Juan JM, Sanz J, González-Casado G. 2019. Assessing the quality of ionospheric models through GNSS positioning error: Methodology and results. GPS Solut 24(1): 4. https://doi.org/10.1007/s10291-019-0918-z.

Sanz J, Juan Zornoza JM, Hernández-Pajares M. 2013. GNSS Data Processing. In: Volume I: Fundamentals and algorithms, ESA Communications, ESTEC, Noordwijk, The Netherlands, pp. 145-161.

Seeber G. 2008. Satellite geodesy: Foundations, methods, and applications, Walter de Gruyter, Berlin, Germany. https://doi.org/ 10.1515/9783110200089.

Talaya J, Bosch E. 1999. CATNET, a permanent GPS network with real-time capabilities. In: Proc. ION GPS-99, 12th Int. Tech. Meeting of The Satellite Division of The US Institute of Navigation, pp. 14-17.

Teunissen PJG. 1995. The least-squares ambiguity decorrelation adjustment: A method for fast GPS integer ambiguity estimation. $J$ Geodesy 70: 65-82.

Wielgosz P, Kashani I, Grejner-Brzezinska D. 2005. Analysis of longrange network RTK during a severe ionospheric storm. $J$ Geodesy 79(9): 524-531. https://doi.org/10.1007/s00190-005-0003-y.

Cite this article as: Timoté CC, Juan JM, Sanz J, González-Casado G, Rovira-García A, et al. 2020. Impact of medium-scale traveling ionospheric disturbances on network real-time kinematic services: CATNET study case. J. Space Weather Space Clim. 10, 29. 